\title{
Networks of microRNAs and genes in acute lymphoblastic leukemia
}

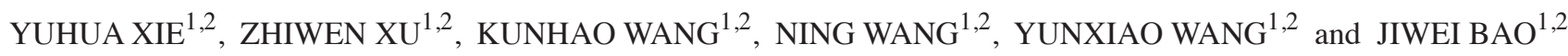 \\ ${ }^{1}$ Department of Computer Science and Technology, Jilin University; \\ ${ }^{2}$ Key Laboratory of Symbolic Computation and Knowledge Engineering of Ministry of Education, \\ Jilin University, Changchun, Jilin 130012, P.R. China
}

Received October 5, 2014; Accepted June 18, 2015

DOI: $10.3892 / \mathrm{mmr} .2015 .4116$

\begin{abstract}
In the present study, three regulatory networks, including a network of differentially expressed factors, a related network and a global network, were constructed hierarchically in order to analyze the association between genes, micro (mi)RNAs and transcriptional factors (TFs) in a systematical approach, rather than focusing on only one or several miRNAs or TFs. By analyzing and comparing the similarities and differences among these three networks, a number of key pathways were highlighted. In addition, identifying the upstream and the downstream nodes, which were composed of differentially expressed genes and miRNAs in the networks provided assistance in identifying associations between circle-regulations or self-adaptation regulations among these elements. In the present study, the TP53 gene and the TP53 pathway were observed to be important in acute lymphoblastic leukemia (ALL). However, the predicted transcriptional factors, including EFKB1 and E2F1, which were found with self-adaptaion associations and certain abnormally expressed miRNAs in the network of differentially expressed factors, requires further examination in further investigations of the pathogenesis of ALL. The confirmation of these factors may be of significance to ALL.
\end{abstract}

\section{Introduction}

Increasing attention has been focussed on investigations into the genes and microRNAs (miRNAs) of acute lymphoblastic

Correspondence to: Professor Zhiwen Xu, Department of Computer Science and Technology, Jilin University, 2699 Qianjin Street, Changchun, Jilin 130012, P.R. China

E-mail: xuzw@jlu.edu.cn

Abbreviations: miRNA, microRNA; TFs, transcription factors; targets, target genes; ALL, acute lymphoblastic leukemia; NCBI, national center for biotechnology information; TFBSs, transcription factor binding sites

Key words: acute lymphoblastic leukemia, microRNA, transcription factors, network, host gene leukemia (ALL). However, elucidation of the pathogenesis of ALL is likely to remain challenging if a comprehensive view is not established in analyzing this disease. ALL is an acute form of leukemia or cancer of the white blood cells, characterized by the overproduction of cancerous, immature white blood cells, termed lymphoblasts $(1,2)$. It is a heterogeneous type of cancer, which is characterized by the rapid and uncontrolled proliferation of immature B- or T-lymphoid precursors. It represents the most common type of childhood malignant neoplasia and, despite significant progress in current treatment methods, 20-30\% of affected children relapse and the causes remain to be elucidated (3).

Transcription factors (TFs) and micro (mi)RNAs are prominent regulators of gene expression (4). TFs are specific proteins, which activate or repress the transcription of genes by binding to $c i s$-regulatory elements located in the upstream regions of genes (5). TFs regulate gene expression either alone or in combination with other proteins at the transcriptional level (6). miRNAs are small, non-coding RNAs, which are pivotal in several cellular functions, including proliferation, differentiation and apoptosis. There are certain genes, which are targeted by miRNAs and there are numerous databases supplying a substantial quantity of experimentally validated data to investigate the associations between miRNAs and their targets (7).

The genes, on which miRNAs are located are termed the host genes of these miRNAs. Rodriguez et al indicated that miRNAs are transcribed in parallel with their host transcripts, and identified two transcription classes of miRNAs, exonic and intronic (8). Baskerville et al indicated that intronic miRNAs and their host genes are closely associated (9). Intronic miRNAs and their host genes are usually coordinately expressed in biological progression. They usually act as a potential partner to achieve biological functions and affect the alteration of pathways (10).

Numerous experiments have been performed to investigate differentially expressed genes and miRNAs, which has enabled a deeper understanding of ALL (11-15). However, in practice, the majority of these experiments focused on a single element, gene or miRNA, which limits the ability to establish the general pathogenesis of ALL. The present study aimed to focus on all associations between genes and miRNAs, including how genes regulate miRNAs, how miRNAs target genes and how 
miRNAs locate on host genes. Each of these elements were analyzed in a comprehensive investigation and focuses on all the ALL-associated elements obtained from databases. The present study then aimed to construct the associated networks, containing the global network, the related network and the network of differentially expressed factors, to determine the differentially expressed genes and miRNAs involved in the pathogenesis of ALL, and the involvement of the global and related networks. By analyzing and comparing the similarities and differences among these networks, a number of key pathways may be highlighted, which may be of significance in the pathogenesis of ALL. In addition, the present study aimed to construct a transcriptional network to enable long-term investigations using predicted transcriptional factors, which may provide a novel direction in future investigations.

\section{Materials and methods}

Material collection and data processing. The experimentally validated dataset of human miRNAs (hsa-mir or hsa-let) and their target genes were obtained from Tarbase 5.0 (http://diana. cslab.ece.ntua.gr/tarbase/) and miRTarBase (http://mirtarbase. mbc.nctu.edu.tw/). The symbols used in the present study to unify each gene and miRNA were the official symbols from the National Center for Biotechnology Information (NCBI) database (http://www.ncbi.nlm.nih.gov/gene/).

A human experimentally-validated dataset of TFs and miRNAs was extracted from TransmiR (http://www.cuilab. cn/transmir) (16), the data of which is obtained from publicly available literature and biological experiments.

The host gene of human miRNA was manually extracted from the miRBase (http://www.mirbase.org/) (17) and NCBI databases. The official symbol and official ID was used to describe each host gene.

The differentially expressed genes, composed of mutated genes, aberrantly-expressed protein, overexpressed genes and single nucleotide polymorphisms (SNPs) were obtained from the Cancer Genetics Web (http://www.cancerindex.org/ geneweb/index.html) and NCBI SNP database (http://www. ncbi.nlm.nih.gov/snp/) and the relevant literature (11-15). The ALL-associated genes were obtained from the GeneCards database (www.genecards.org) (18) and relevant literature, which included genes affecting tumor growth and migration, and genes involved in radiation therapy, prevention, diagnosis, development and the clinical outcome of ALL. The differentially-expressed genes, mentioned above, were also considered to be a part of the associated genes. In addition, 28 important TFs were extracted using the P-match method. Briefly, 1,000 nt promoter region sequences of the targets of differentially expressed genes were downloaded from the UCSC database (19), and the P-match method, which combines pattern matching and weight matrix approaches, was used to identify transcription factor binding sites (TFBSs) in the $1,000 \mathrm{nt}$ promoter region sequences. The TFBSs were then mapped onto the promoter region of targets. The matrix library of P-match also contains sets of known TFBSs, which are collected in TRANSFAC (http:/www.biobase-international.com/), providing the possibility to identify a variety of TF binding sites. The vertebrate matrix was used with restricted high-quality criterion for the matrix. This method enables the identification of the possible TFs that may have effects in ALL. In the present study, only human elements were selected, and the corresponding genes were determined, which were also considered to be associated-genes. However, the TFs examined in the study were only those that appeared in transmiR.

Subsequently, the differentially-expressed miRNAs were extracted from the mir2Disease database (http://www. mir2disease.org/) (20) and relevant literature. The ALL miRNAs were obtained manually from the relevant literature, and the differentially expressed miRNAs were added to these.

Construction of the networks. All the regulatory associations between the genes regulating miRNAs, miRNA target genes and miRNA location on the host genes, were analyzed. Following combining all these associations, Ctyoscype software version 3.1.1 (U.S. National Institute of General Medical Sciences; award number GM070743) was used to construct the global network. The network of differentially expressed factors was extracted from the global network, however, only the associations between the differentially expressed genes the and miRNAs with their host genes were selected from the global network. Following combining of these associations, the network of differentially expressed factors was constructed. The same method was used to constructing the ALL-related network.

\section{Results}

Association between differentially expressed factors of ALL. The significant regulatory association between differentially expressed genes and miRNAs in ALL is shown in Fig. 1. The network is comprised of mirRNAs and their host genes, five TFs (TP53, TF3, RUNX1, LMO2 and BCR) and other genes, which were aberrantly expressed in ALL and targeted by at least one differentially expressed miRNA. In terms of the functions of the TFs, certain important pathways were identified, for example, the mir-222-TP53-mir155 and the TCF3-mir451-BCL2 pathways were observed in the differentially-expressed network, and they may represent a deeper association between genes and miRNAs. Although, there is no specific data to indicate a direct association between miRNAs and miRNAs, they may affect on each other in an indirect manner.

For example, since miR-142 is regulated by LOM2, and LOM2 is targeted by miR-223, it was suggested that hsa-mir-223 affects has-mir-142 indirectly via LOM2. In addition, genes were observed to have similar associations, for example TF3 regulates miR-451 directly, while has-mir-451 targets the BCL2 gene. Therefore, it was hypothesized that there may be an association between TCF3 and BCL2, with hsa-mir-451 as a transition. It was also observed that miRNAs were regulated by more than one $\mathrm{TF}$, and that the genes may be targeted by several miRNAs. For example, miR-27a was observed to be regulated by RUNX1 while TP53 and BCR exhibited co-regulation with miR-155. In addition, ATM was found to be targeted by two miRNAs (miR-101 and miR-100) simultaneously, BCL2 was targeted by four miRNAs and ABL1 was only targeted by miR-203. As for the miRNAs and their host genes, although the host genes themselves were 


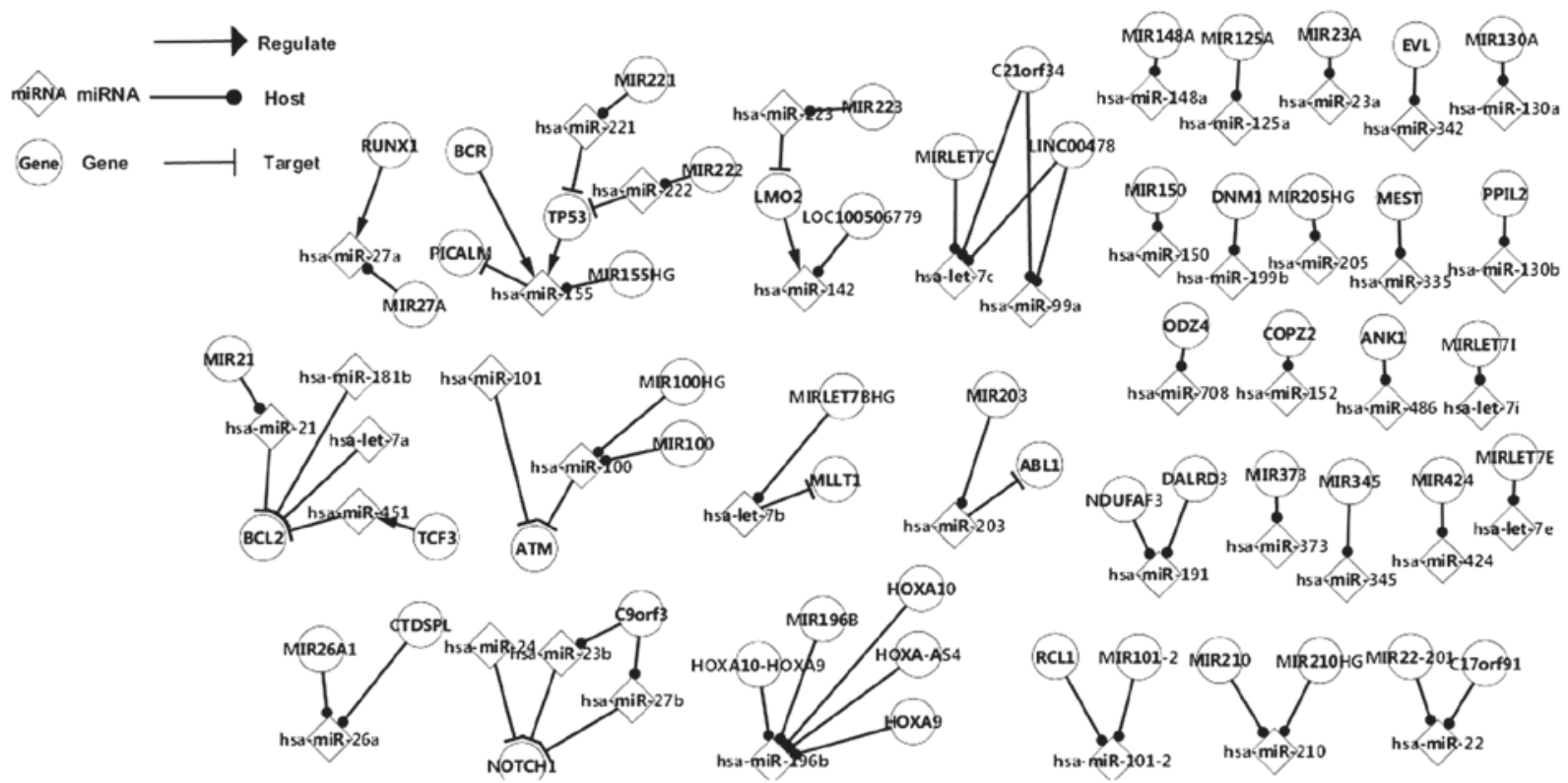

Figure 1. Associations (regulation, targets and hosts) between differentially expressed genes and miRNAs in acute lymphoblastic leukemia. miRNA/miR, microRNA.

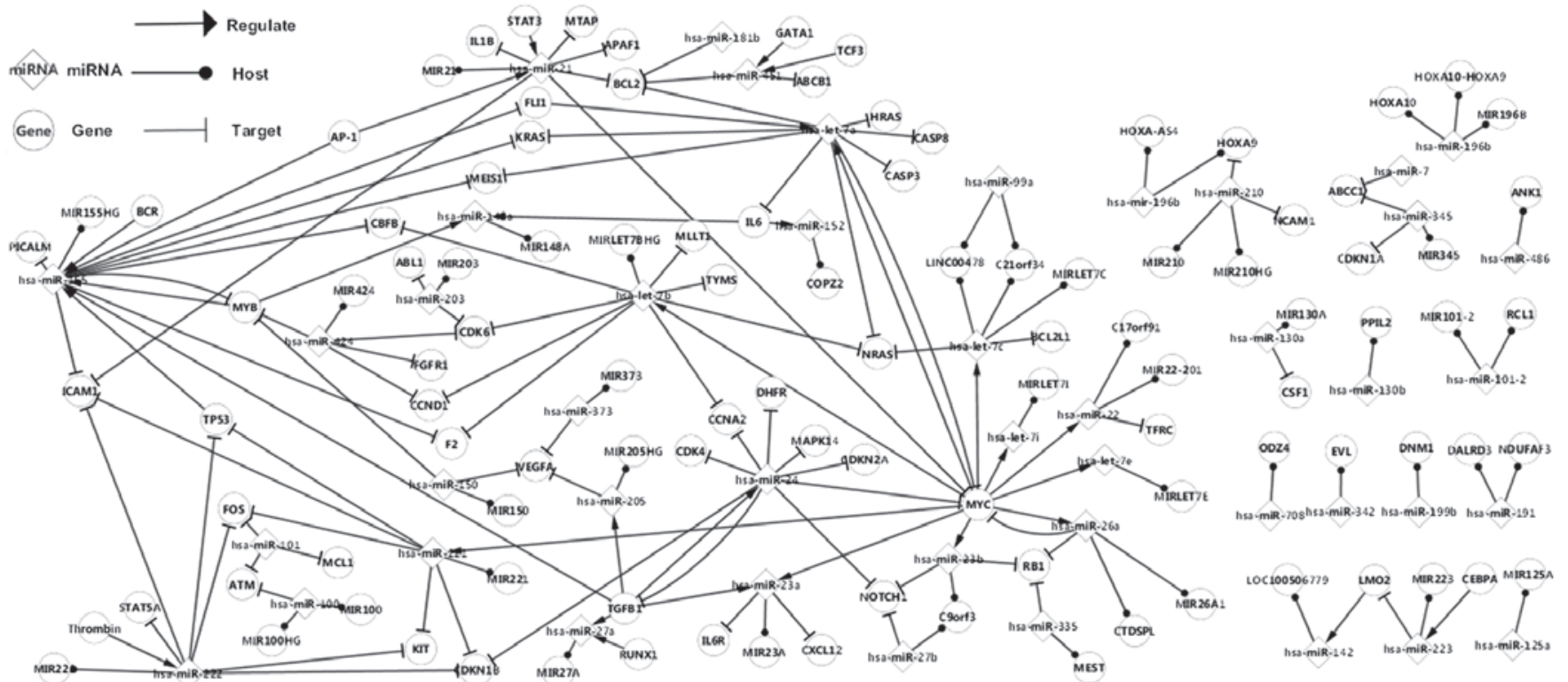

Figure 2. Networks of associated genes and microRNAs in acute lymphoblastic leukemia.

not differentially-expressed in ALL, their miRNAs were aberrantly expressed. The C9orf3 contained hsa-mir-23b and hsa-mir-27b, which targeted NOTCH1 with hsa-mir-24, and C21orf34 and LINC00478 contained hsa-mir-99a and hsa-let-7c, whilst hsa-let-7c located on the MIRLET7C gene. Several of the differently expressed miRNAs in ALL were found to locate to only one gene, including miR-708 or miR-502. However, exceptions included hsa-mir-101-2, which located to RCL1 and MIR101-2, while hsa-mir-196 was found on 5 host genes in total.

The identification of additional genes and miRNAs in the network of differentially expressed factors is likely in future studies of this disease, however, the network of differentially expressed factors established in the present study identifies and offers insight into the regulatory pathogenesis of ALL.

Network of genes and miRNAs involved in ALL. The second network constructed (Fig. 2) represents a more complex association, compared with the network of differentially expressed factors, since it contains a larger number of genes and miRNAs, and more comprehensive pathways. In particular, the differentially expressed genes and miRNAs are included in this network. As a result, this network of differentially expressed factors forms the related network. In the present study, certain specific pathways were observed in this network. A self-adaptation association was identified between MYC and 


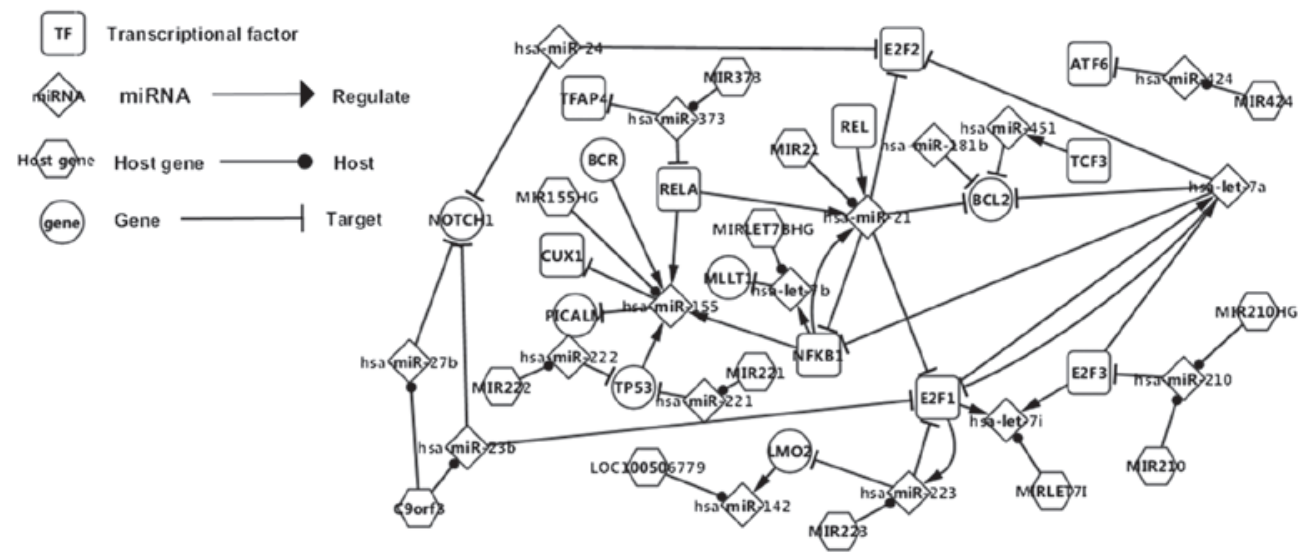

Figure 3. Network of predicted transcription factors and differentially expressed miRNAs and genes in acute lymphoblastic leukemia. miRNA, microRNA.

has-let-7a, MYC and hsa-mir-26b, TGFB1 and hsa-mir-24, and MYB and hsa-mir-255, meaning that these genes may have an effect on themselves via the miRNAs they regulate.

The related network elucidated additional topological associations of differentially expressed elements, for instance, the network of the TP53 was immensely expanded with the addition of the relative nodes, contributing to the understanding of the progression of ALL.

Regulation by predicted TFs and differentially expressed genes and miRNAs in ALL. The regulatory network of predicted TFs, differentially expressed genes and miRNAs, termed the global network, in ALL covers more comprehensive regulatory associations between these three datasets. It is an experimentally validated biological network in the human body, and it contains all the confirmed genes, miRNAs and associations between humans. Notably, it is too complex to present in the current study. Additionaly, it includes the network of differentially expressed factors and the related network.

Transcriptional network of predicted TFs. A total of 14 differentially expressed miRNAs, which were regulated by predicted TFs or targeted these TFs, identified in the transcriptional network, were analyzed further. Fig. 3 shows the regulatory associations of predicted TFs and the differentially expressed miRNAs in ALL. These elements sequentially affect their successors by targeting or regulating them. The differentially expressed genes, which were observed to be indirectly or directly associated with the predicted TFs were also included in the transcription network. For the predicted TFs, the p-match method was used to identify genes, which were predicted to be involved in the process of ALL, a number of which have already been confirmed as differentially expressed, including TCF3 and PAX5 (21). The TFs were combined with the differentially expressed genes and mirRNAs, as well as their host genes, to obtain an overall view of how TFs affect the differentially expressed miRNAs. The transcriptional network revealed a potential mechanism involved in the pathogenesis of ALL, and requires further investigation in the future. Determination of whether the associations among the network are involved in ALL may assist in further investigations aiming to cure the disease. For example, a self-adaptation association between hsa-miR-223 and E2F1, which exhibits the same association with hsa-let-7a, was observed (Fig. 3). Therefore, E2F1 may be involved in ALL for the special position it has with the differentially expressed miRNAs and the predicted associations require confirmation. Similar associations were also identified in NFKB1.

Regulation by differentially expressed genes. The upstream and downstream factors of the elements identified as important were also identified in the present study, including the differentially expressed genes, differentially expressed miRNAs and the TFs obtained using P-match method, in order to examine the network of ALL more clearly.

In addition, the successor and precursor nodes of the differentially expressed genes in the three networks were extracted in order to analyze and compare the regulatory pathways. Among these genes, TP53 and LMO2 may exhibit the typical characteristics with their precursor and successor nodes, indicating the specific regulatory association between the gene and miRNAs, and the associations between the miRNAs.

The data presented in Table I shows TP53 and the precursors and successors of the gene, as well as their regulatory associations. TP53 was significantly featured in the three networks. hsa-miR-221 and hsa-miR-222 were observed to target TP53, which itself regulates hsa-miR-155 in these three networks (Table I). Therefore, the hsa-miR-221 and hsa-miR-222 miRNAs indirectly affect hsa-miR-155 through TP53. A mutation in the TP53 gene may be implicated in the pathogenesis of ALL via constitutive activation of the TP53 pathway.

Regulation by differentially expressed microRNAs. The same method used to compare and analyze differentially expressed genes was also applied to the differentially expressed miRNA pathways, which had been previously extracted. Of all these miRNAs, three miRNAs, including hsa-miR-27a, hsa-miR-155 and hsa-miR-451, with their corresponding genes, were observed to exhibit a specific association between miRNAs and genes, as well as associations between themselves.

Table II presents the data of hsa-miR-155 and its predecessor and successor nodes, which were extracted from the three networks. The results (Table II) indicated that TP53 and BCR regulate hsa-miR-155, which targeted PICALM in the 
Table I. Regulatory associations between miRNAs and the TP53 gene in the three networks.

\begin{tabular}{llr}
\hline $\begin{array}{l}\text { Differentially- } \\
\text { expressed miRNA }\end{array}$ & $\begin{array}{l}\text { Related } \\
\text { network }\end{array}$ & $\begin{array}{r}\text { Global } \\
\text { network }\end{array}$ \\
\hline
\end{tabular}

Targeting TP53

hsa-miR-221

hsa-miR-222

Regulated by TP53

hsa-miR-155

\section{hsa-miR-221}

hsa-miR-222

hsa-miR-155
hsa-miR-125b-1

hsa-miR-125b

hsa-miR-125b-2

hsa-miR-1285

hsa-miR-1285-1

hsa-miR-1285-2

hsa-miR-15a

hsa-miR-16

hsa-miR-16-1

hsa-miR-16-2

hsa-miR-221

hsa-miR-222

hsa-miR-25

hsa-miR-30d

hsa-miR-612

hsa-miR-107
Table I. Continued.

\begin{tabular}{llr}
\hline $\begin{array}{l}\text { Differentially- } \\
\text { expressed network }\end{array}$ & $\begin{array}{l}\text { Related } \\
\text { network }\end{array}$ & $\begin{array}{r}\text { Global } \\
\text { network }\end{array}$ \\
\hline & $\begin{array}{l}\text { hsa-miR-34c } \\
\text { hsa-miR-519d }\end{array}$ \\
\hline miR, microRNA. & \\
\hline
\end{tabular}

hsa-miR-125b

hsa-miR-125b-1

hsa-miR-125b-2

hsa-miR-143

hsa-miR-145

hsa-miR-155

hsa-miR-192

hsa-miR-194

hsa-miR-194-1

hsa-miR-194-2

hsa-miR-200a

hsa-miR-200b

hsa-miR-200c

hsa-miR-215

hsa-miR-29

hsa-miR-29a

hsa-miR-29b-1

hsa-miR-29b-2

hsa-miR-29c

hsa-miR-34

hsa-miR-34a

hsa-miR-34b

hsa-miR-34c

hsa-miR-519d

hsa-miR-29a

hsa-miR-29b-1

hsa-miR-29b-2

hsa-miR-29c

hsa-miR-34

hsa-miR-34a

hsa-miR-34b
Table II. Regulatory associations between genes and hsa-miR-155 in the three networks.

\begin{tabular}{llc}
\hline $\begin{array}{l}\text { Differentially- } \\
\text { expressed genes }\end{array}$ & $\begin{array}{l}\text { Related } \\
\text { network }\end{array}$ & $\begin{array}{c}\text { Global } \\
\text { network }\end{array}$ \\
\hline
\end{tabular}

\begin{tabular}{|c|c|c|}
\hline \multicolumn{3}{|c|}{ Targeting hsa-miR-155 } \\
\hline TP53 & MYB & IRF1 \\
\hline \multirow[t]{13}{*}{ BCR } & TGFB1 & JUN \\
\hline & TP53 & MYB \\
\hline & AP-1 & NFKB1 \\
\hline & BCR & RELA \\
\hline & & SMAD4 \\
\hline & & SMG1 \\
\hline & & SPI1 \\
\hline & & TGFB1 \\
\hline & & TP53 \\
\hline & & AKT1 \\
\hline & & BCR \\
\hline & & CAMP \\
\hline & & FOXP3 \\
\hline \multicolumn{3}{|c|}{ Regulated by hsa-miR-155 } \\
\hline \multirow[t]{23}{*}{ PICALM } & CBFB & AGTR1 \\
\hline & F2 & AMIGO2 \\
\hline & FLI1 & ANKFY1 \\
\hline & ICAM1 & APC \\
\hline & KRAS & ARFIP1 \\
\hline & MEIS1 & ARFIP2 \\
\hline & MYB & ARID2 \\
\hline & PICALM & ARL10 \\
\hline & & ARL5B \\
\hline & & ATG3 \\
\hline & & ATP6V1C1 \\
\hline & & BACH1 \\
\hline & & BCAT1 \\
\hline & & BET1 \\
\hline & & BRPF3 \\
\hline & & C3orf58 \\
\hline & & C5orf41 \\
\hline & & CAMTA1 \\
\hline & & CBFB \\
\hline & & CDK5RAP3 \\
\hline & & CEBPB \\
\hline & & CHAF1A \\
\hline & & C5orf41 \\
\hline
\end{tabular}


Table II. Continued.

\begin{tabular}{lll}
\hline $\begin{array}{l}\text { Differentially- } \\
\text { expressed genes }\end{array}$ & $\begin{array}{l}\text { Related } \\
\text { network }\end{array}$ & $\begin{array}{r}\text { Global } \\
\text { network }\end{array}$ \\
\hline & CAMTA1 \\
& CBFB \\
& CDK5RAP3 \\
& CEBPB \\
& CHAF1A \\
\hline
\end{tabular}

miR, microRNA.

Table III. Regulatory associations between miRNAs and the NFKB1 gene.

\begin{tabular}{llr}
\hline $\begin{array}{l}\text { Differentially- } \\
\text { expressed miRNA }\end{array}$ & $\begin{array}{l}\text { Related } \\
\text { network }\end{array}$ & $\begin{array}{r}\text { Global } \\
\text { network }\end{array}$ \\
\hline
\end{tabular}

\section{Targeting NFKB1}

hsa-let-7a

hsa-miR-21

$\begin{array}{ll}\text { hsa-let-7a } & \text { hsa-let-7a } \\ \text { hsa-miR-21 } & \text { hsa-let-7a-1 } \\ & \text { hsa-let-7a-2 } \\ & \text { hsa-let-7a-3 } \\ & \text { hsa-miR-146a } \\ & \text { hsa-miR-146b } \\ & \text { hsa-miR-15a } \\ & \text { hsa-miR-9 } \\ & \text { hsa-miR-9-1 } \\ & \text { hsa-miR-9-2 } \\ & \text { hsa-miR-9-3 }\end{array}$

Regulated by NFKB1

hsa-let-7b

hsa-let-7b

hsa-miR-155

hsa-miR-21
hsa-miR-155

hsa-miR-21 hsa-let-7a-3

hsa-let-7b

hsa-miR-10b
Table III. Continued

\begin{tabular}{|c|c|c|}
\hline $\begin{array}{l}\text { Differentially- } \\
\text { expressed miRNA }\end{array}$ & $\begin{array}{l}\text { Related } \\
\text { network }\end{array}$ & $\begin{array}{l}\text { Global } \\
\text { network }\end{array}$ \\
\hline & & $\begin{array}{l}\text { hsa-miR-21 } \\
\text { hsa-miR-365 } \\
\text { hsa-miR-365-1 } \\
\text { hsa-miR-365-2 } \\
\text { hsa-miR-365a } \\
\text { hsa-miR-365b } \\
\text { hsa-miR-448 } \\
\text { hsa-miR-9 } \\
\text { hsa-miR-91 } \\
\text { hsa-miR-9-1 } \\
\text { hsa-miR-9-2 } \\
\text { hsa-miR-9-3 } \\
\text { hsa-miR-199a-2 } \\
\text { hsa-miR-214 } \\
\text { hsa-miR-224 } \\
\text { hsa-miR-29a } \\
\text { hsa-miR-29b } \\
\text { hsa-miR-29b-1 } \\
\text { hsa-miR-29b-2 } \\
\text { hsa-miR-29c } \\
\text { hsa-miR-34 } \\
\text { hsa-miR-34a } \\
\text { hsa-miR-365 } \\
\text { hsa-miR-365-1 } \\
\text { hsa-miR-365-2 } \\
\text { hsa-miR-365a } \\
\text { hsa-miR-365b } \\
\text { hsa-miR-448 } \\
\text { hsa-miR-9 } \\
\text { hsa-miR-91 } \\
\text { hsa-miR-9-1 } \\
\text { hsa-miR-9-2 } \\
\text { hsa-miR-9-3 }\end{array}$ \\
\hline
\end{tabular}

hsa-miR-125b

hsa-miR-125b-1

hsa-miR-125b-2

hsa-miR-146a

hsa-miR-155

hsa-miR-16

hsa-miR-16-1

hsa-miR-16-2

hsa-miR-17

hsa-miR-199a-2

hsa-miR-21

hsa-miR-214

hsa-miR-224

hsa-miR-29a

hsa-miR-29b

hsa-miR-29b-1

hsa-miR-29b-2

hsa-miR-29c

hsa-miR-34

hsa-miR-34a

\begin{tabular}{ll}
\hline $\begin{array}{l}\text { Differentially- } \\
\text { expressed miRNA }\end{array}$ & \multicolumn{1}{c}{$\begin{array}{c}\text { Global } \\
\text { network }\end{array}$} \\
\hline network
\end{tabular}

network of differentially expressed factors, which indicate that TP53 and BCR may either co-regulate or individually regulate PICALM indirectly by hsa-miR-155. It was also demonstrated that MYB and hsa-miR-155 form self-adaptation associations separately.

Regulation by popular TFs. With the same method used for the differentially expressed miRNAs, data of the predicted TFs data, using the P-match method, were processed. The precursor and successor nodes from the three networks were extracted, classed and listed. Specific regulatory associations were highlighted in the list, two predicted TFs, E2F1 and NFKB1 exhibited an important characteristic, which was common to the precursor and successor nodes among these TFs, as self-adaption associations were identified between 
these TFs and their corresponding miRNAs. For example, four differentially expressed miRNAs, including hsa-let-7a, hsa-miR-21, hsa-miR-223 and hsa-miR-23b, were observed to target E2F1, and three differentially expressed miRNAs, including hsa-let-7a, hsa-miR-223 and hsa-let-7i, were regulated by E2F2. Furthermore, the hsa-let-7a and hsa-miR-223 miRNAs formed separate self-adaption associations with E2F1.

The predicted TFs in a global network were subsequently analyzed, and all the TFs were classified into four categories. The first class has six types of adjacent nodes, comprising three types of precursor and three types of successor. TFs, including E2F1, E2F3, NFKB1 and RELA belonged to this class.

The data in Table III is that of NFKB1, which contains the precursor and successor elements of the network of differentially expressed factors, the related network and the global network. The data demonstrated that hsa-miR-21 separately forms a self-adaption association with NFKB1. Notably, no mutation of NFKB1 in ALL has been reported previously, to the best of our knowledge. As hsa-miR-21 was identified from the network of differentially expressed factors, this miRNA may affect the aberrant expression of other miRNAs through NFKB1.

The second class of TFs were those TFs, which contained only three types of precursors or successors. E2F2, REL, CUX1, TFAP4, TCF3 and ATF6 were identified in this class.

The third class of TFs had only one adjacent node, either a precursor or successor, and included CREB1 and STAT1.

The final class of TFs had neither a precursor node nor a successor, and contained E2F4, NR2F2, HLF, ZEB, ELK1, PAX5, RORA, ZBTB6, RREB1 and the ATF family (ATF1-5 and 7). The reason for the identification of TFs with no adjacent nodes may be due to the limited quantity of data that can be obtained to analyze ALL. This indicates that additional experimentally validated data are required and may offer a new direction in investigating ALL.

\section{Discussion}

The present study constructed and examined the network of differentially expressed factors and the transcription network of predicted transcription factors. In the present study, pathways contain three or more elements were identified, for example, hsa-miR-221 targets TP53, and TP53 regulates hsa-miR-155, which targets PICALM. The TCF3 pathway was also observed to contain three elements (TCF3, hsa-miR451 and BCL2). These pathways may have a biological function in ALL.

TP53 has been identified as a typical gene that is abnormally expressed in other types of cancer, including retinoblastoma and human Hodgkin's lymphoma, which suggests that TP53 requires attention in further investigations of ALL, and may assist in defining the similarities among types of cancer. The resulting understanding of the associations between genes may be extended from one type of carcinoma to another, and a systematic view in analyzing diseases may be obtained.

Another area, which requires further investigation is the TFs, which were identified using the P-match method, and suggested a potential association between the differentially expressed miRNAs and TFs. These predicted associations require experimental validation to improve understanding of the pathogenic mechanism of ALL.

The present study constructed three regulatory topological networks of the genes, miRNAs involved in ALL, and data were extracted from the network in order to highlight specific pathways, genes and transcription factors, which may be important in the investigation of ALL. Focus on the successors and precursors of the nodes in these three networks may assist in analyzing the network. In addition, construction of a network using predictions with the P-match method may providing important data for the further investigation of ALL.

\section{Acknowledgements}

This study was supported by grants from the National Natural Science Foundation of China (grant. no. 60973091) and the Science and Technology Development Plan of Jilin Province (grant. no. 20130101166JC).

\section{References}

1. Weinblatt ME: Pediatric acute myelocytic leukemia. In: Sakamoto KM, Windle ML, Cripe TP, Arceci RJ (eds). Medscape Reference. WebMD. Accessed 17 April 2014.

2. Seiter K: Acute lymphoblastic leukemia. In: Sarkodee-Adoo C Talavera F, Sacher RA, Besa EC (eds). Medscape Reference. WebMD. Accessed 17 April 2014.

3. Pui CH: Recent research advances in childhood acute lymphoblastic leukemia. J Formos Med Assoc 109: 777-787, 2010.

4. Hobert O: Gene regulation by transcription factors and microRNAs. Science 319: 1785-1786, 2008.

5. Tran DH, Satou K, Ho TB and Pham TH: Computational discovery of miR-TF regulatory modules in human genome. Bioinformation 4: 371-377, 2010.

6. Martin KJ: The interactions of transcription factors and their adaptors, coactivators and accessory proteins. BioEssays 13: 499-503, 1991.

7. Zhang BH; Pan XP; Cobb GP, et al: Plant microRNA: A small regulatory molecule with big impact. Dev Biol 289: 3-16, 2006.

8. Rodriguez A, Griffiths-Jones S, Ashurst JL and Bradley A: Identification of mammalian microRNA host genes and transcription units. Genome Res 14: 1902-1910, 2004.

9. Baskerville $\mathrm{S}$ and Bartel D: Microarray profiling of microRNAs reveals frequent coexpression with neighboring miRNAs and host genes. RNA 11: 241-247, 2005.

10. Ramalingam P, Palanichamy JK, Singh A, Das P, Bhagat M, Kassab MA, Sinha S and Chattopadhyay P: Biogenesis of intronic miRNAs located in clusters by independent transcription and alternative splicing. RNA 20: 76-87, 2014.

11. Armstrong SA, Mabon ME, Silverman LB, et al: FLT3 mutations in childhood acute lymphoblastic leukemia. Blood 103: 35443546, 2004.

12. Tartaglia M, Martinelli S, Cazzaniga G, et al: The PTPN11 gene is mutated in pediatric acute lymphoblastic leukemia. Blood 102: 65A-65A, 2003.

13. Grossmann V, Haferlach C, Weissmann S, et al: The molecular profile of adult T-cell acute lymphoblastic leukemia: Mutations in RUNX1 and DNMT3A are associated with poor prognosis in T-ALL. Genes Chromosomes Cancer 52: 410-422, 2013.

14. Zenatti PP, Ribeiro D, Li W, Zuurbier L, Silva MC, Paganin M, Tritapoe J, Hixon JA, Silveira AB, Cardoso BA, et al: Oncogenic IL7R gain-of-function mutations in childhood T-cell acute lymphoblastic leukemia. Nat Genet 43: 932-939, 2011.

15. Malyukova A, Dohda T, von der Lehr N, Akhoondi S, Corcoran M, Heyman M, Spruck C, Grandér D, Lendahl U and Sangfelt O. The tumor suppressor gene hCDC4 is frequently mutated in human T-cell acute lymphoblastic leukemia with functional consequences for Notch signaling. Cancer Res 15: 5611-5616, 2008.

16. Wang J, Lu M, Qiu C and Cui Q: TransmiR: A transcription factor-microRNA regulation database. Nucleic Acids Res 38: D119-D122, 2010. 
17. Kozomara A and Griffiths-Jones S: miRBase: Integrating microRNA annotation and deep-sequencing data. Nucleic Acids Res 39: D152-D157, 2011.

18. Safran M, Dalah I, Alexander J, Rosen N, Iny Stein T, Shmoish M, Nativ N, Bahir I, Doniger T, Krug H, et al: GeneCards Version3: The human gene integrator. Database (Oxford) 2010: baq020, 2010.

19. Fujita PA, Rhead B, Zweig AS, Hinrichs AS, Karolchik D, Cline MS, Goldman M, Barber GP, Clawson H, Coelho A, et al: The UCSC Genome Browser database: Update 2011. Nucleic Acids Res 39: D876-D882, 2011.
20. Bao J, Li D, Wang L, et al: MicroRNA-449 and microRNA-34b/c function redundantly in murine testes by targeting E2F transcription factor-retinoblastoma protein $(\mathrm{E} 2 \mathrm{~F}-\mathrm{pRb})$ pathway. J Biol Chem 287: 21686-21698, 2012.

21. Familiades J, Bousquet M, Lafage-Pochitaloff M, et al: PAX5 mutations occur frequently in adult B-cell progenitor acute lymphoblastic leukemia and PAX5 haploinsufficiency is associated with BCR-ABL1 and TCF3-PBX1 fusion genes: A GRAALL study. Leukemia 23: 1989-1998, 2009. 